intervention impact (relative reduction in MSM HIV prevalence after 10 years: A: $18.2 \%$ (95\% CI 11.0 to $29.2 \%$ ), B: $18.1 \%$ (10.6 to $29.7 \%)$ ). Conclusions The choice of method used to balance insertive and receptive contacts in an HIV transmission model affected the estimates for the amount of like-with-like mixing within different MSM groups, but the estimated impact of an intervention was robust to the method used.

\section{P1-S4.25 USING MATHEMATICAL MODELS TO UNDERSTAND THE CAUSES OF THE ECOLOGICAL ASSOCIATION SEEN BETWEEN HIV AND HSV-2 IN FEMALE SEX WORKERS IN SOUTHERN INDIA}

doi:10.1136/sextrans-2011-050108.169

${ }^{1} \mathrm{~K}$ Mitchell, ${ }^{1} \mathrm{P}$ Vickerman, ${ }^{2} \mathrm{M}$ Pickles, ${ }^{2} \mathrm{M}$ Kaushik, ${ }^{3} \mathrm{~S}$ Verma, ${ }^{3} \mathrm{~S}$ Isac, ${ }^{4} \mathrm{R}$ Adhikary, ${ }^{5} \mathrm{M}$ Mainkar, ${ }^{6} \mathrm{M}$ Alary, ${ }^{2} \mathrm{M} \mathrm{C}$ Boily. ${ }^{1}$ London School of Hygiene and Tropical Medicine, London, UK; ${ }^{2}$ Imperial College London, London, UK; ${ }^{3}$ Karnataka Health Promotion Trust, Bangalore, India; ${ }^{4}$ Family Health International, New Delhi, India; ${ }^{5}$ National AIDS Research Institute, Pune, India; ${ }^{6}$ Centre Hospitalier Affilié Universitaire de Québec, Québec, Canada

Background Cross-sectional surveys of female sex workers (FSW) in multiple districts in Southern India show a strong ecological association between HIV and HSV-2 prevalence. Modelling was used to determine if this association is primarily due to shared behavioural risk factors (such as partner change rate) or if biological interactions between the two viruses also play a major role.

Methods Linear regression was used to find significant predictors of FSW HIV prevalence in 17 districts in Southern India (using data from Avahan, the India AIDS Initiative). Explanatory variables investigated included prevalence of different STIs in FSW and clients, mean duration of being an FSW or client, and mean reported number of clients per FSW or FSW visited per client. A deterministic HIV/HSV-2 transmission model without behavioural heterogeneity or transmission co-factors was parameterised using district-specific FSW and client behavioural data and fitted to HIV and HSV-2 prevalence data for each district by varying the per partnership HIV and HSV-2 transmission probabilities. The model was firstly fit to all districts simultaneously assuming a constant HIV and HSV-2 transmission probability to see whether district-level variations in behaviour were sufficient to explain the trends seen, and then fit separately for each district to test for trends in the estimated transmission parameters in different settings.

Results FSW HSV-2, syphilis, gonorrhoea and chlamydia prevalence, client HIV prevalence and mean number of clients per FSW per week

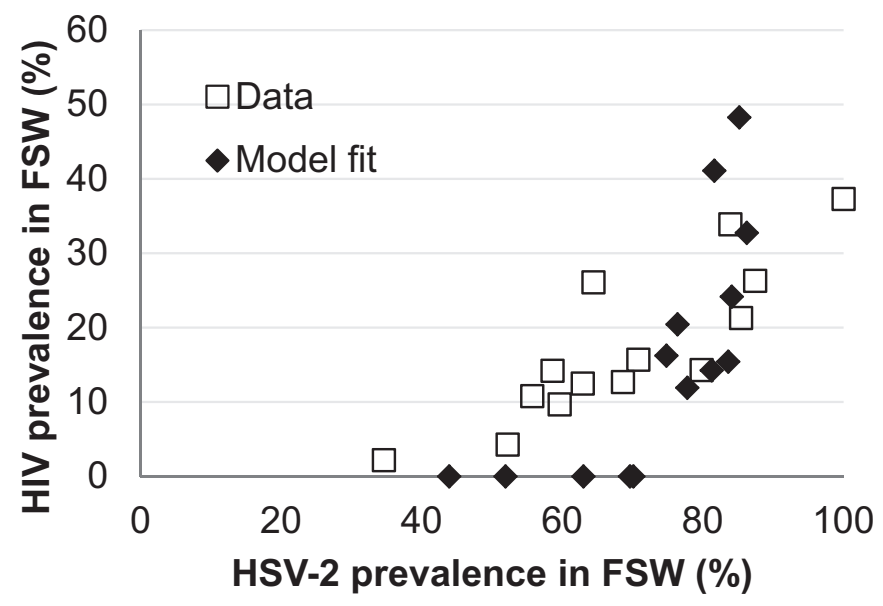

Abstract P1-S4.25 Figure 1 FSW. were all correlated with FSW HIV prevalence, but only FSW HSV-2 prevalence remained a significant predictor in multivariate analysis. The dynamic model reproduced the HIV/HSV-2 association when simultaneously fit to all districts, but was a poor fit to data (Abstract P1-S4.25 figure 1). When individual transmission probabilities were fit for each district positive correlations were seen between the HSV-2 transmission probability and both the HIV transmission probability and HSV-2 prevalence, but not between the HIV transmission probability and HSV-2 prevalence or vice versa.

Conclusion These results suggest that differences in mean reported partner change rate or duration of commercial sex are not sufficient to explain the association between HIV and HSV-2 prevalence in FSW in Southern India. However, initial analyses do not show clear evidence for a biological interaction. More detailed models will be used to further investigate the association.

\section{P1-S4.26 DURATION, INCIDENCE AND PREVALENCE OF CHLAMYDIA TRACHOMATIS IN WOMEN: ESTIMATION BY MULTI-PARAMETER SYNTHESIS}

doi:10.1136/sextrans-2011-050108.170

${ }^{1} \mathrm{M}$ Price, ${ }^{1} \mathrm{~A} \mathrm{E}$ Ades, ${ }^{2} \mathrm{D}$ De Angelis, ${ }^{1} \mathrm{~N}$ Welton, ${ }^{1} \mathrm{~J}$ Macleod, ${ }^{3} \mathrm{~K}$ Soldan, ${ }^{1} \mathrm{~K}$ Turner ${ }^{1} \mathrm{I}$ Simms, ${ }^{1} \mathrm{P}$ Horner. ${ }^{1}$ University of Bristol, Bristol, UK; ${ }^{2}$ Cambridge University, UK; ${ }^{3}$ Health Protection Agency, UK

Background An understanding of the prevalence and incidence of Chlamydia trachomatis (CT) infection is needed to assess the potential value of screening. Typically, the estimation of incidence, prevalence and duration are seen as distinct exercises. Here we estimate them simultaneously from the available data subject to the wellknown relationship prevalence $=$ incidence times duration.

Methods We re-examine studies of duration of asymptomatic CT, based on recent reviews, and propose a model. Information from a recent synthesis of UK prevalence studies, and data on infection and re-infection rates in UK clinic settings, are used to generate estimates of incidence of infection in the general population, taking account of the effect of duration of infection on observed incidence. We use Bayesian multi-parameter evidence synthesis to check the consistency of the evidence and to produce internally coherent estimates of duration, incidence and prevalence in women.

Results The three sets of evidence sources that directly inform incidence, prevalence and duration respectively were consistent with each other. Our estimates are: duration of asymptomatic infection 1.25 years $(1.04,1.50)$, average incidence and prevalence in $16-44$ year olds $2.2 \%(1.7,2.9)$ per year and $2.1 \%(1.7,2.6)$ respectively.

Conclusions The apparently heterogeneous estimates of duration of asymptomatic CT in the literature are explained by the different study designs. Adapted appropriately, they agree with UK prevalence and incidence data.

\section{Epidemiology poster session 4: Tests evaluation}

\section{P1-S4.27 OUALITY ASSURANCE OF SYPHILIS SEROLOGICAL TEST IN GUANGDONG, CHINA, 2004-2009}

doi:10.1136/sextrans-2011-050108.171

H P Zheng, X Z Wu, JinM Huang. Guangdong Provincial Center for Skin Diseases and STls Control, Guangzhou, China

Background Syphilis has made a major comeback in China, now representing the most common communicable disease in many cities and regions. A total of 327433 cases of syphilis were reported 
in 2009, accounting for a sevenfold increase since 1999. Accurate treponemal and non-treponemal serological testing are critical to providing a correct diagnosis, but there are substantial variations in the quality of serological diagnostic tests and testing across different parts of China. Since 2003, the Guangdong Provincial STI Control Center in collaboration with the Bureau of Public Health has developed a program on STIs laboratory construction and quality assurance system. We report the proficiency of serological test of syphilis among the STIs laboratories in Guangdong Province in 2004-2009.

Methods Proficiency panels consisting of five samples with syphilis positive and negative sera were prepared in the provincial laboratory and sent to STIs laboratories for non-treponemal and treponemal testing once a year. Participating laboratories were asked to report the type of test used and quantitative and qualitative results for each serum. Each quantitative result was compared to the geometric mean of all participants' results, and was considered correct if it was less than fourfold difference from the mean titre.

Results The numbers of STIs laboratories in Guangdong which participated in the survey increased from 19 to 225 and a total of 13203 sera were tested from 2004 to 2009. 98\% laboratories used the "toluidine red unheated serum" (TRUST) as their non-treponemal test and all laboratories used the "Treponema pallidum particle agglutination assay" (TPPA) as their treponemal test. The mean accuracies of non-treponemal tests among the laboratories evaluated increased significantly from $78.9 \%$ in 2004 to $97.7 \%$ in 2009 $\left(\chi^{2}=17.11, \mathrm{p}<0.01\right)$ for qualitative test results, and from $75.8 \%$ in 2004 to $90.8 \%$ in $2009\left(\chi^{2}=8.09, p<0.01\right)$ for quantitative test results. Higher accuracies were observed with TPPA ranging from 93.5 to $100 \%\left(\chi^{2}=2.85, \mathrm{p}>0.05\right)$ for qualitative test results and improved from $73.7 \%$ to $86.5 \%\left(\chi^{2}=4.7, p<0.05\right)$ for quantitative test results during this period. For non-treponemal testing, $4.1 \%$ $(113 / 2754)$ of the results were found to be false negative and $2.3 \%$ $(34 / 1456)$ were found to be false positive. For qualitative treponemal results, $6.5 \%(96 / 1470)$ and $1.9 \%(17 / 889)$ of treponemal results were found to be false-negative and false-positive respectively. For quantitative results, it was worth noting that in some cases, four to seven-titre deviations were observed for the same sera.

Conclusion This province-wide STIs laboratory construction and quality assurance system has helped to improve the accuracies of serological syphilis testing over time. But there is still room for improvement to facilitate improved control of syphilis in China.

\section{P1-S4.28 SURVEY OF METHODOLOGY USED FOR THE IDENTIFICATION AND ANTIMICROBIAL SUSCEPTIBILITY TESTING OF NEISSERIA GONORRHOEAE IN LATIN AMERICA AND THE CARIBBEAN}

doi:10.1136/sextrans-2011-050108.172

S Starnino, M Liao, M Ruben, A Storey, J A R Dillon, GASP-LAC Network*. Vaccine and Infectious Disease Organization, University of Saskatchewan, Saskatoon, Canada

Background The Gonococcal Antimicrobial Susceptibility Surveillance Program in Latin America and the Caribbean (GASP-LAC) in the 1990s had significant impact in identifying trends in antimicrobial susceptibility in the region. To revitalise the GASP-LAC, a survey was undertaken to determine the level of surveillance activity and the methods used for the identification and antimicrobial susceptibility testing (AST) of Neisseria gonorrhoeae (Ng) isolates.

Methods A structured questionnaire was distributed to potential participants to collect information regarding surveillance activities and methods used for identification and AST of $\mathrm{Ng}$ in LAC countries. Information was also obtained from presentations at the Workshop of the GASP-LAC in November 2010 in Buenos Aires, Argentina.
Results 7 countries completed the questionnaire and four provided unstructured answers regarding the methodologies. All 11 countries were interested in continuing to participate in the GASP-LAC. Of nine countries reporting, seven had an on-going country-wide network for gonococcal AST and two countries collected isolates locally, the number of isolates tested each year varied (25-400). Thayer Martin medium was used for $\mathrm{Ng}$ primary culture by all countries answering this question $(n=8)$; among them, four countries used biochemical tests alone, or coupled with Gram stain $(n=3)$ and one country, in addition to the two methods, used antigen detection and the nucleic acid amplification method. Chromogenic cephalosporin was used by all respondents $(n=9)$ for detecting $B$ lactamase production. Methods used for AST included agar dilution in 6 of 9 reporting countries, coupled with disc diffusion $(n=4)$ and Etest $(n=2)$; the remaining used disc diffusion alone $(n=1)$ or coupled with Etest $(n=2)$. CLSI interpretation criteria were used in all responding participants $(n=9)$. $\mathrm{Ng}$ reference strains included ATCC49226 $(n=6)$, coupled with WHO III, V, VII $(n=2)$ or WHO A$\mathrm{E}(\mathrm{n}=1)$.

Conclusions Different levels of surveillance were noted between countries probably due to various resource availabilities. On the basis of these responses the GASP-LAC Co-ordinating Centre will reestablish and consolidate the GASP-LAC.

*Authors contributed equally and are listed in an alphabetical order of country names. P Galarza, I Pagano, M E Trigoso, A Schwartz Benzaken, V M Pinto, A Maldonado Ballesteros, 0 M. Sanabria Cruz, A Llop, E Aguilar Jarrin, N Aguayo, J L Portilla Carbajal, G Borthagaray, A Acevedo, D Payares

\section{P1-S4.29 SYNTHESIS OF EVIDENCE ON IMPLEMENTATION RESEARCH ON POINT-OF-CARE SYPHILIS TESTS: A SYSTEMATIC REVIEW}

doi:10.1136/sextrans-2011-050108.173

${ }^{1} \mathrm{Y}$ Jafari, ${ }^{2} \mathrm{M}$ Johri, ${ }^{3} \mathrm{D}$ Ako-Arrey, ${ }^{1} \mathrm{~S}$ Shivkumar, ${ }^{4} \mathrm{G}$ Lambert, ${ }^{5} \mathrm{C}$ Claessens, ${ }^{1} \mathrm{M}$ Klein, ${ }^{6} \mathrm{~J}$ Cajas, ${ }^{7} \mathrm{R}$ Peeling, ${ }^{1} \mathrm{~N}$ Pai. ${ }^{1}$ McGill University, Montreal, Canada; ${ }^{2}$ Université de Montreal, Canada; ${ }^{3}$ University of Saskatchewan, Canada; ${ }^{4}$ Institut national de santé publique du Québec, Canada; ${ }^{5}$ Laboratoire de santé publique du Québec, INSPO, Canada; ${ }^{6}$ Queen's University, Canada; ' London School of Hygiene \& Tropical Medicine, UK

Background With the increase in global prevalence of syphilis, synthesis of evidence of point-of-care (POC) assays is warranted. While a clear methodology exists to meta-analyse diagnostic performance, a clear rubric that incorporates implementation research outcomes (IRO) relevant for policy making is lacking. Recently, Grading of Recommendations Assessment, Development and Evaluation (GRADE) working group called for a shift to emphasis on patient-centred outcomes for making policy recommendations. However, a lack of clarity in defining, elucidating, and reporting of these outcomes prevents their utilisation in practice. Within this context, we reviewed global evidence on IROs for syphilis POC tests.

Method We systematically searched nine electronic databases for the period of January 1980 to September 2010. Articles that addressed IRO regarding POC syphilis tests were reviewed and data extracted. A second reviewer independently reviewed a subset of the articles. Outcomes were synthesised into a narrative review.

Results 31 (48\%) from 64 full text articles assessed were included in the narrative review. Twenty-four studies were cross-sectional, six were case-control, while one was a clustered randomised control trial (RCT). IROs were categorised into: Acceptability, Preference, Feasibility, Prevalence, Barriers and Challenges, and Economic Evaluations of POC tests. Three papers reported outcomes on acceptability, four on preference, ten on feasibility, seven on impact, six on prevalence, seven on barriers and challenges, and seven on economic 